Supporting Information Section.

\title{
Transformation of $\alpha$-Tocopherol (Vitamin E) and Related Chromanol Model Compounds into Their Phenoxonium Ions By Chemical Oxidation with the Nitrosonium $\left(\mathrm{NO}^{+}\right)$Cation.
}

\author{
Stephen B. Lee, Ching Yeh Lin, Peter M. W. Gill and Richard D. Webster* \\ Research School of Chemistry, Australian National University, Canberra ACT 0200, Australia \\ "E-mail:webster@rsc.anu.edu.au, Fax: +61261250750
}

\section{CONTENTS}

Page S2. Figure S1. Photograph of $\mathrm{CH}_{3} \mathrm{CN}$ solution containing $\left(\mathrm{CH}_{3}\right) \alpha-\mathrm{TO}^{+}$at $233 \mathrm{~K}$.

Page S3. Figure S2. Solution phase ATR-FTIR spectra obtained during the chemical oxidation of 2,6-di- tert-butyl-4-methoxyphenol in $\mathrm{CH}_{3} \mathrm{CN}$ at $233 \mathrm{~K}$.

Page S4. Figure S3. Solid state FTIR spectra of $(\mathrm{COOH}) \alpha-\mathrm{TO}^{+}$.

Page S5. Figure S4. Theoretical normal modes of vibration of $\left(\mathrm{CH}_{3}\right) \alpha-\mathrm{TO}^{+}$that are infrared active between $1800-1600 \mathrm{~cm}^{-1}$.

Page S6. Figure S5. ${ }^{1} \mathrm{H}$ NMR spectra illustrating decomposition of $\left(\mathrm{CH}_{3}\right) \alpha-\mathrm{TO}^{+}$.

Page S7. Figure S6. Theoretical ${ }^{13} \mathrm{C}$ NMR spectra of $\left(\mathrm{CH}_{3}\right) \alpha-\mathrm{TOH}$ and $\left(\mathrm{CH}_{3}\right) \alpha-\mathrm{TO}^{+}$.

Page S8. Figure S7. EPR spectra of cation radicals produced by the chemical oxidation of phenols in $\mathrm{CH}_{2} \mathrm{Cl}_{2}$.

Page S9-S14. Summary of results from theoretical calculations (Cartesian coordinates, \# of imaginary frequencies and total energies). 


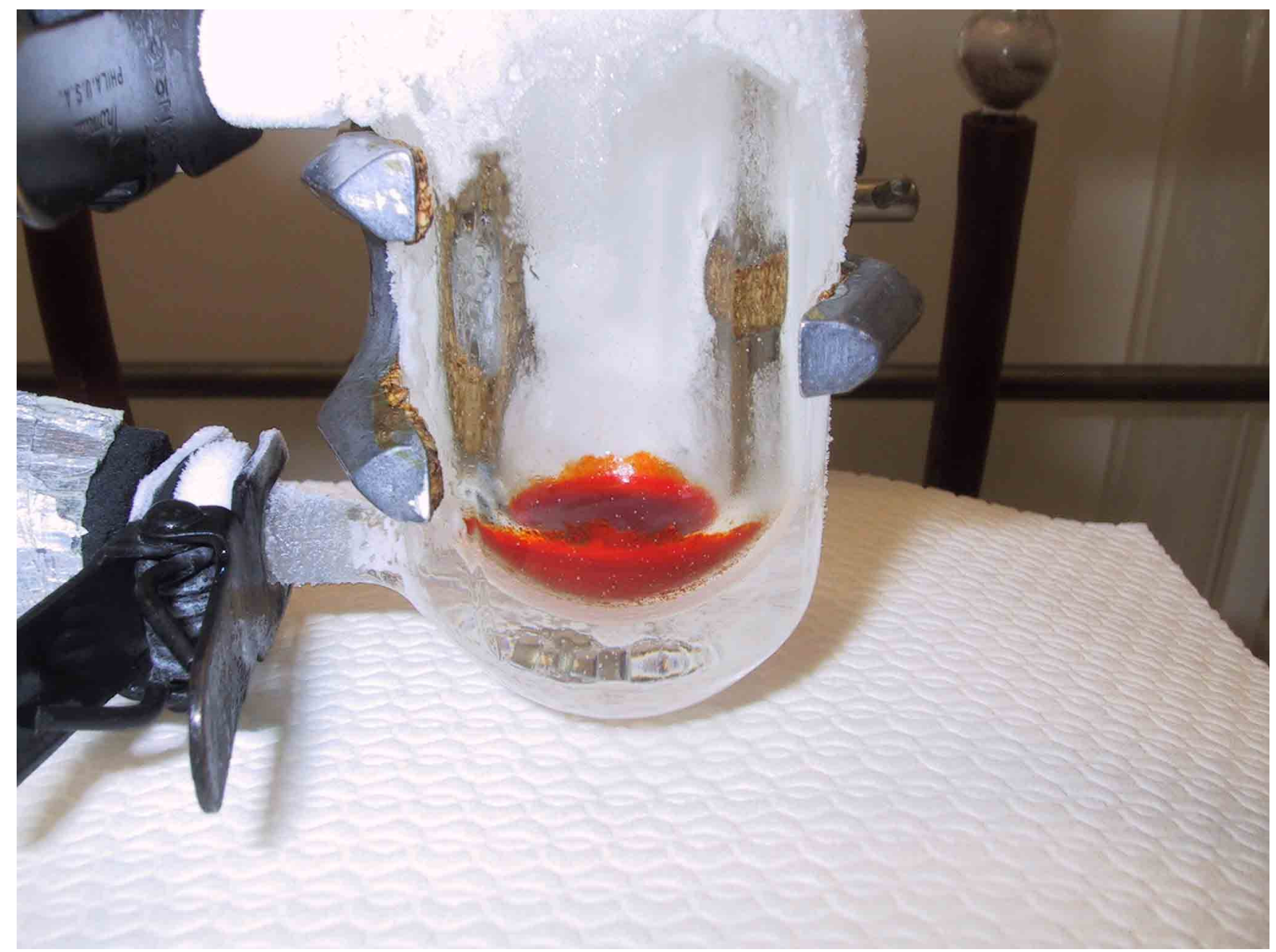

Figure S1. Photograph of $0.57 \mathrm{M}\left(\mathrm{CH}_{3}\right) \alpha-\mathrm{TO}^{+}$in $\mathrm{CH}_{3} \mathrm{CN}$ at $233 \mathrm{~K}$ showing typical bright orange/red color of phenoxonium cations. 


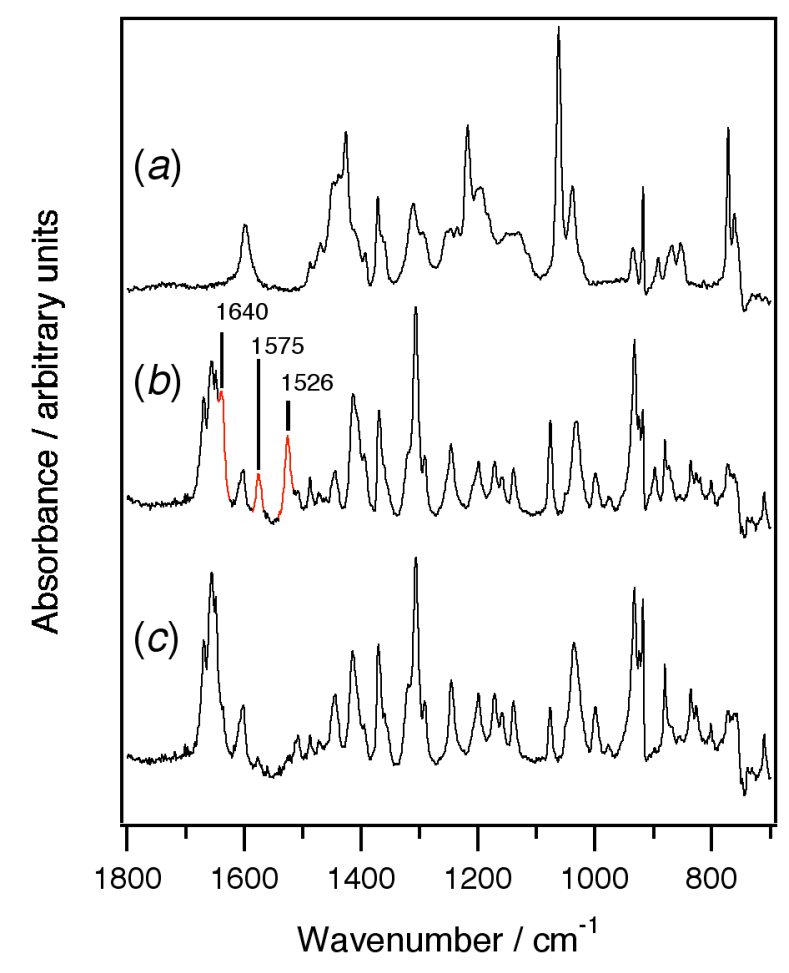

Figure S2. Solution phase ATR-FTIR spectra of $40 \mathrm{mM}$ 2,6-di-tert-butyl-4-methoxyphenol obtained at $233 \mathrm{~K}$ in $\mathrm{CH}_{3} \mathrm{CN}$. (a) Initial spectrum. (b) Two minutes after the addition of 2 mols of $\mathrm{NOSbF}_{6}$. (c) Five minutes after the addition of 2 mols of $\mathrm{NOSbF}_{6}$. Each spectrum represents 128 accumulated scans recorded at $2 \mathrm{~cm}^{-1}$ resolutions. (-) Possible absorbancies due to phenoxonium cation. 


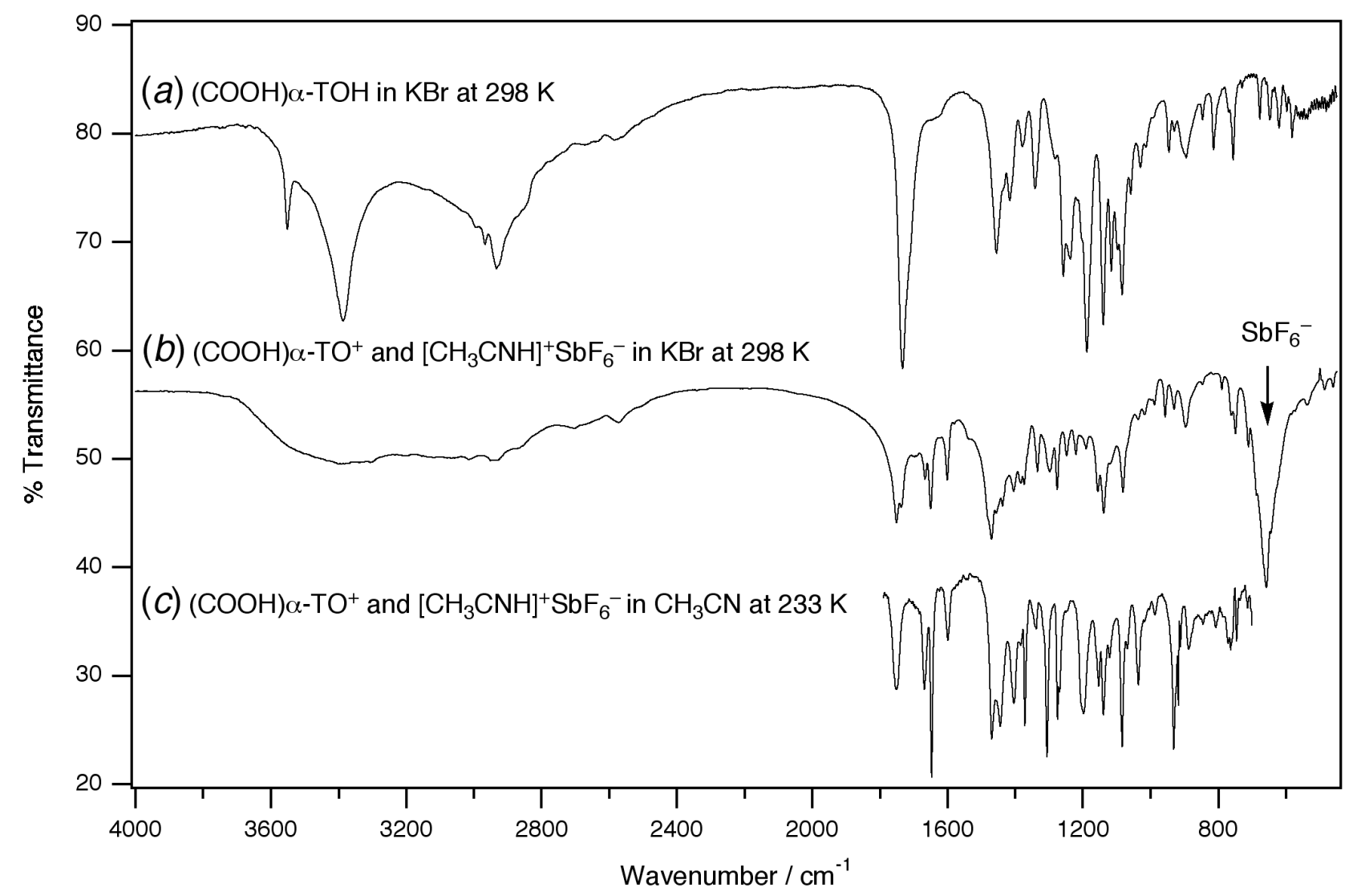

Figure S3. Solid state $(\mathrm{KBr})$ and solution phase $\left(\mathrm{CH}_{3} \mathrm{CN}\right)$ FTIR spectra of $(\mathrm{COOH}) \alpha-\mathrm{TOH}$ and $(\mathrm{COOH}) \alpha-\mathrm{TO}^{+}$. 

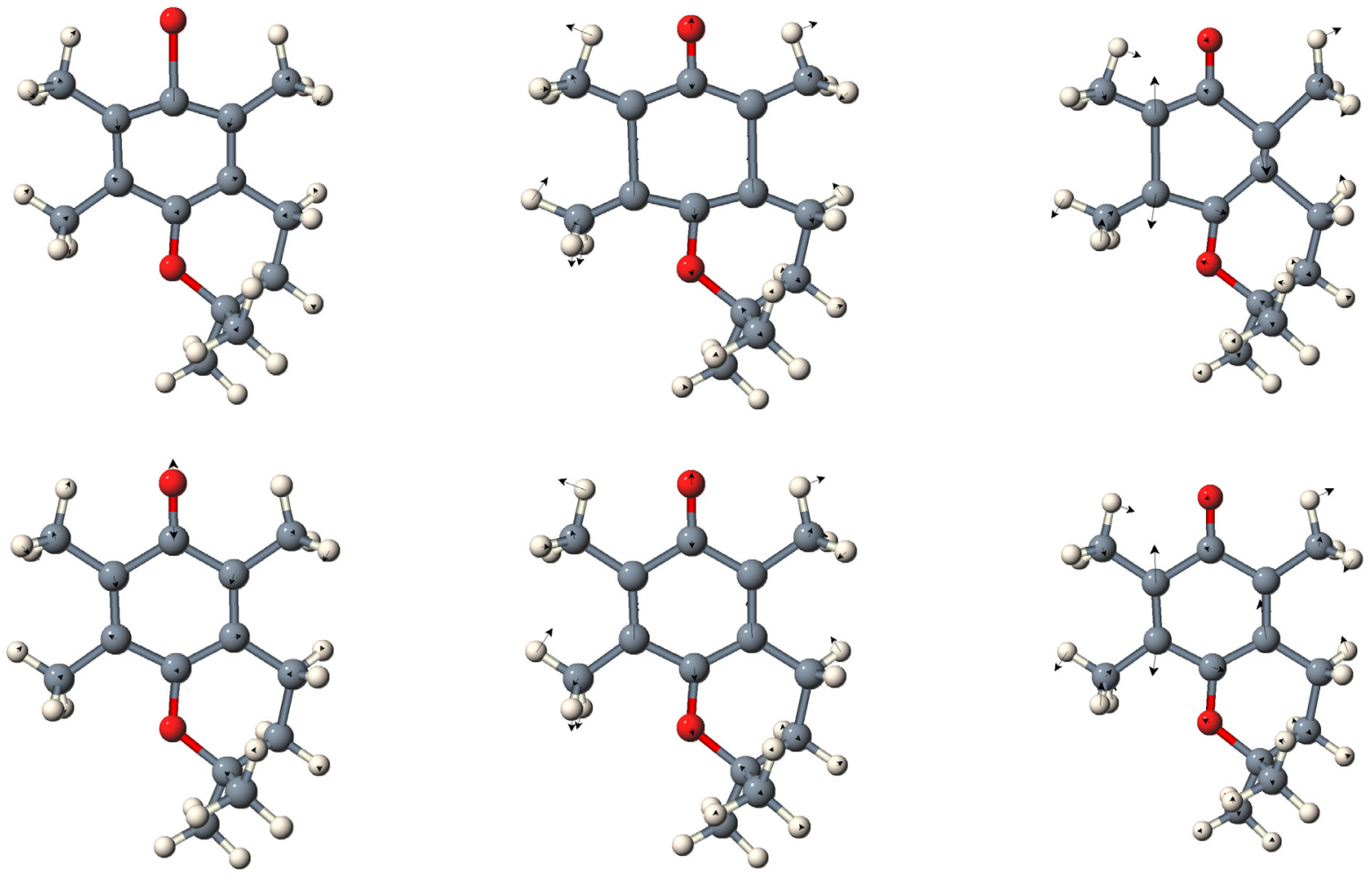

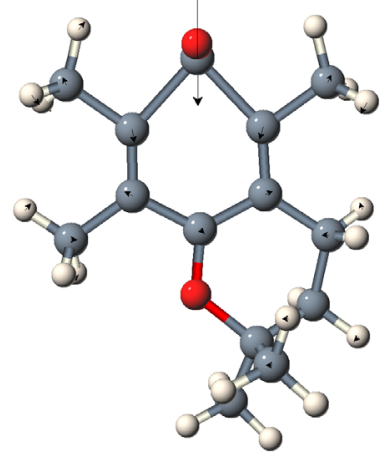

$\mathrm{C}=\mathrm{O}$ stretch

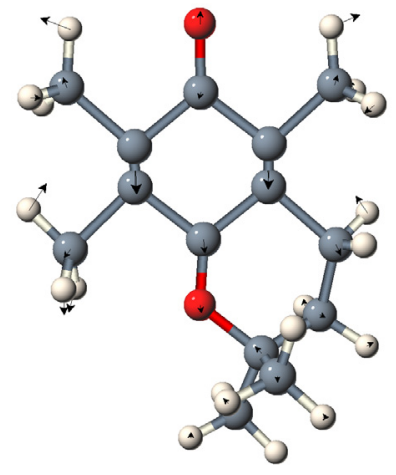

$\mathrm{C}=\mathrm{C}$ ring symmetric stretch

$$
1696 \mathrm{~cm}^{-1}
$$

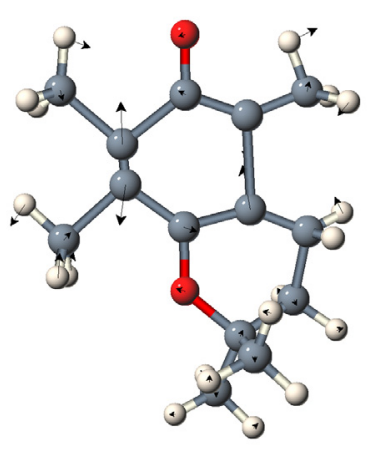

$\mathrm{C}=\mathrm{C}$ ring asymmetric stretch $1638 \mathrm{~cm}^{-1}$

Figure S4. Theoretical normal modes of vibration of $\left(\mathrm{CH}_{3}\right) \alpha-\mathrm{TO}^{+}$active between $1800-1600 \mathrm{~cm}^{-1}$ (Figure 3b). The vibrations correspond to the absorbancies observed at 1670,1649 and $1605 \mathrm{~cm}^{-1}$ in the experimental spectra (Figure 3a). 


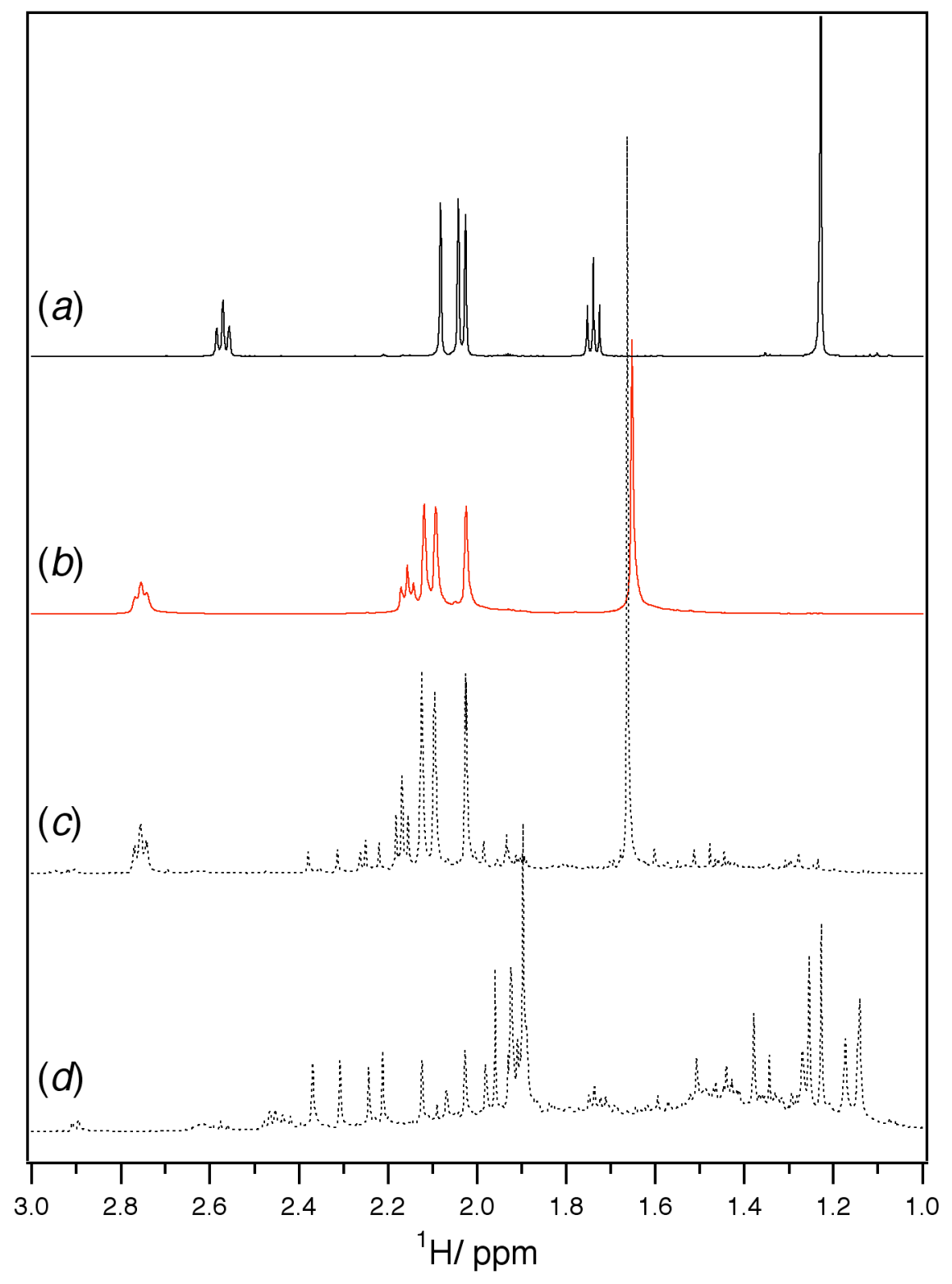

Figure S5. $500 \mathrm{MHz}{ }^{1} \mathrm{H}$ NMR spectra obtained during the oxidation of $0.57 \mathrm{M}\left(\mathrm{CH}_{3}\right) \alpha-\mathrm{TOH}$ in $\mathrm{CD}_{3} \mathrm{CN}$. (a) At $233 \mathrm{~K}$ prior to oxidation. (b) At $233 \mathrm{~K}$ after the addition of 2 mols of $\mathrm{NOSbF}_{6}$ to form $\left(\mathrm{CH}_{3}\right) \alpha-\mathrm{TO}^{+}$. (c) After solution in (b) was warmed to $298 \mathrm{~K}$ for one hour. (d) Immediately after the addition of $1 \mathrm{M} \mathrm{H}_{2} \mathrm{O}$ to the solution in $(c)$. 


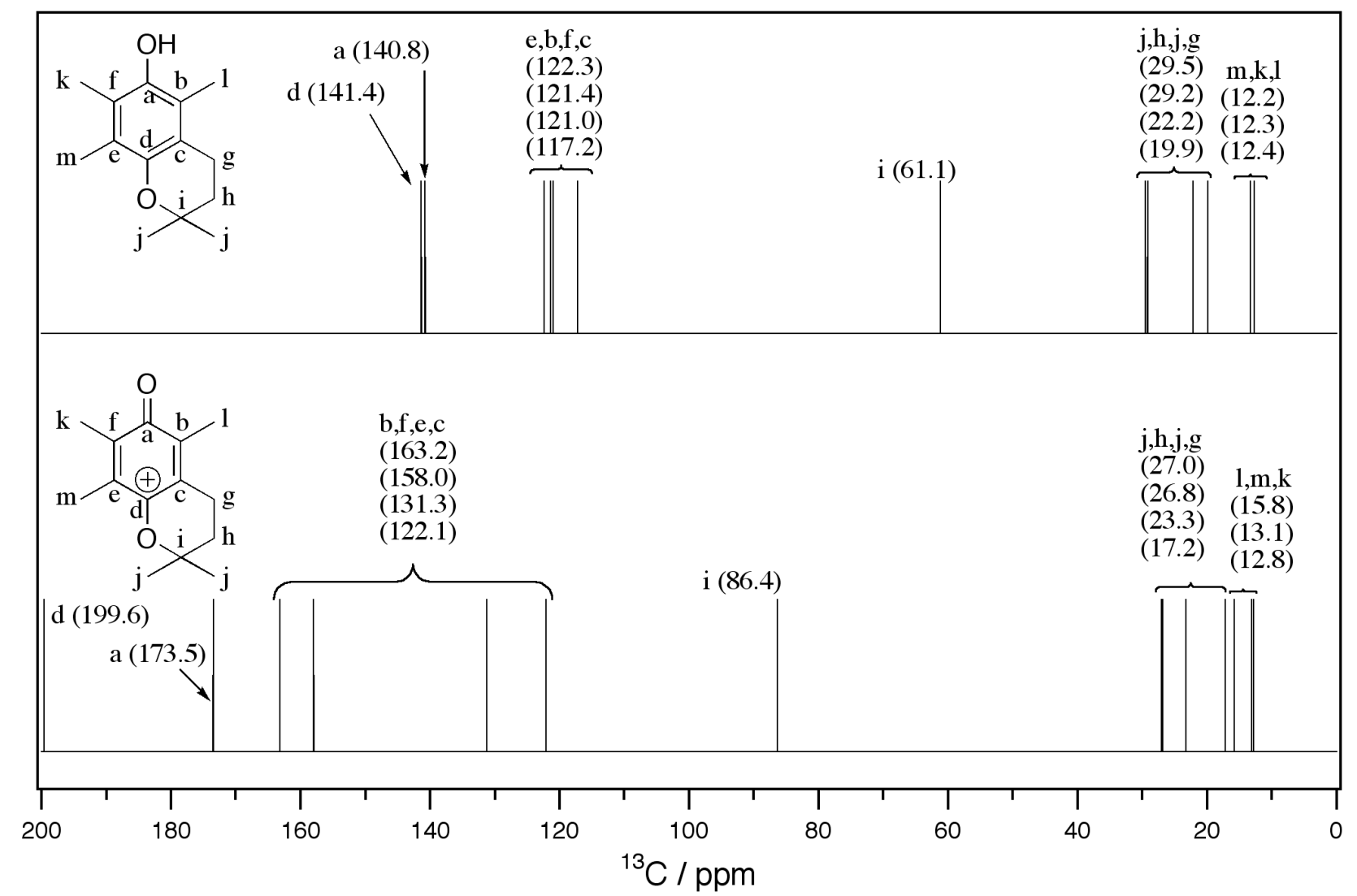

Figure S6. Calculated HF/6-31G* ${ }^{13} \mathrm{C}$ NMR chemical shifts. The two methyl carbons in position $\mathrm{j}$ are non-equivalent on the theoretical time-scale; hence two ${ }^{13} \mathrm{C}$ NMR peaks are predicted. Signal intensities are arbitrary. 


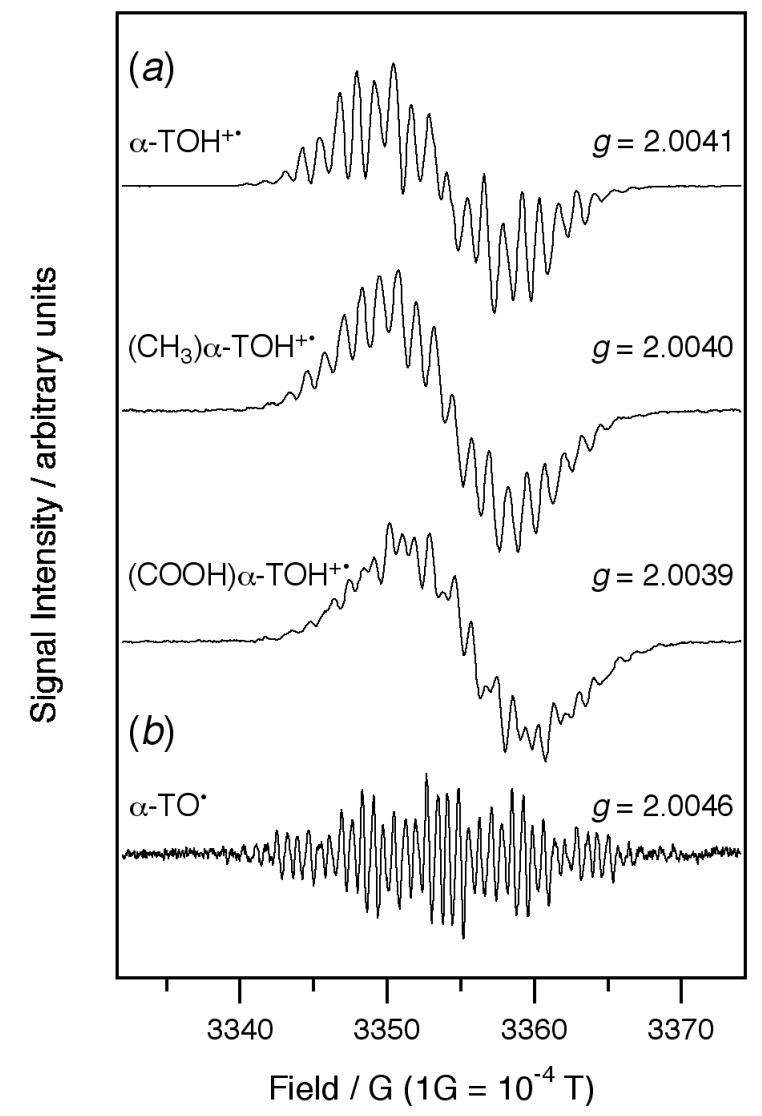

Figure S7. Continuous wave X-band EPR spectra of: (a) Phenol cation radicals obtained by the oxidation of $5 \mathrm{mM}$ of the parent phenols at $223 \mathrm{~K}_{\text {in }} \mathrm{CH}_{2} \mathrm{Cl}_{2}$ with $\mathrm{NOSbF}_{6}$. (b) The neutral phenoxyl radical of vitamin $\mathrm{E}$ generated by one-electron in situ electrochemical oxidation of $2 \mathrm{mM}$ the phenolate anion in $\mathrm{CH}_{3} \mathrm{CN}$ with $0.2 \mathrm{M} \mathrm{Bu}_{4} \mathrm{NPF}_{6}$ at $293 \mathrm{~K}^{.5 b}$ The modulation amplitude $=0.2 \mathrm{G}$, time constant $=$ $0.16 \mathrm{~s}$ and sweep time $=100 \mathrm{~s}$. 
Pages S9-S14. Summary of results from theoretical calculations (Cartesian coordinates, \# of imaginary frequencies and total energies).

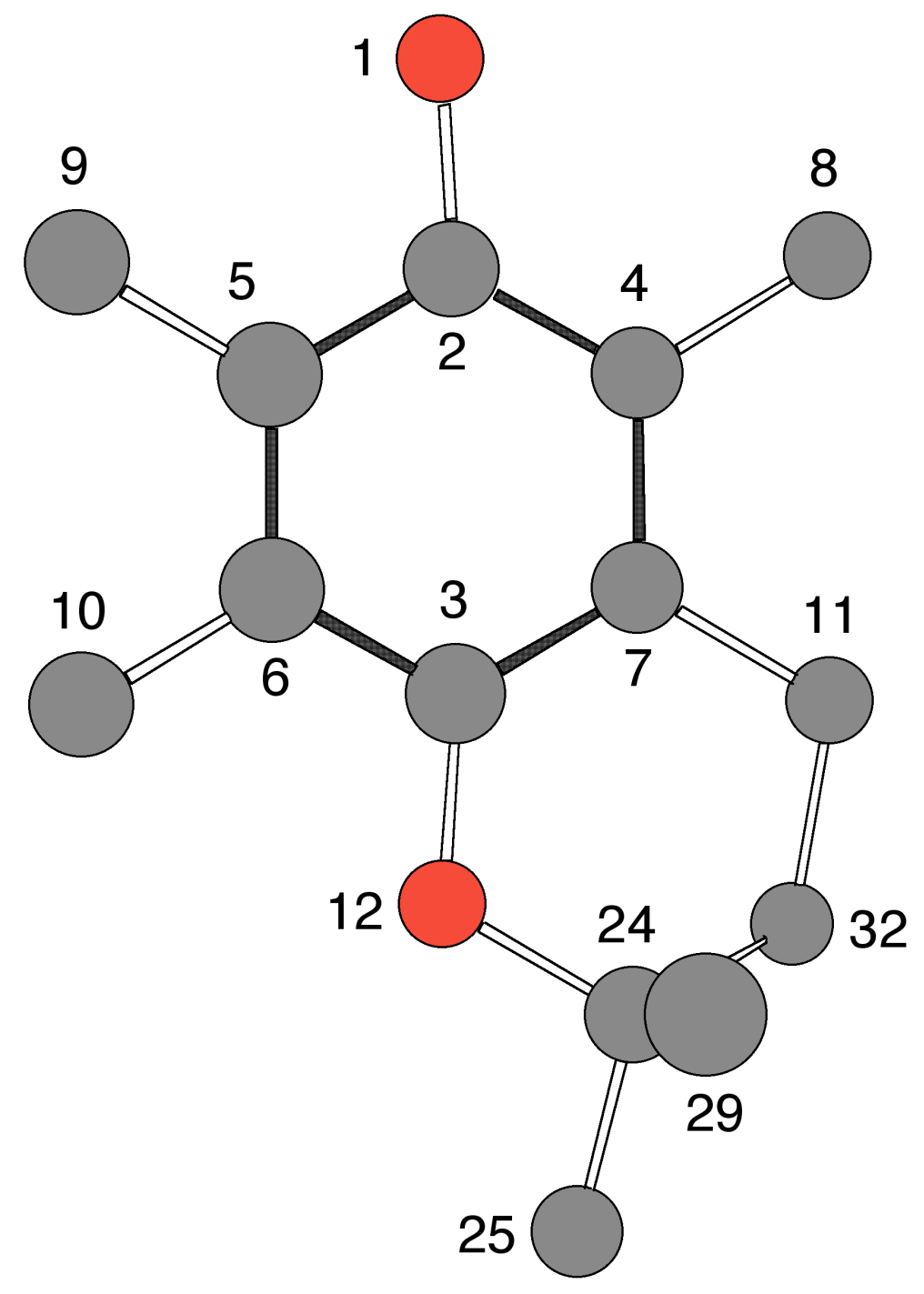

Numbering system for starting material $\left[\left(\mathrm{CH}_{3}\right) \alpha-\mathrm{TOH}\right]$ and phenoxonium cation $\left[\left(\mathrm{CH}_{3}\right) \alpha-\mathrm{TO}^{+}\right]$. Hydrogen atoms are omitted for clarity. Methyl carbons 25 and 29 are non-equivalent on the theoretical time-scale. 


\section{1) $\left(\mathrm{CH}_{3}\right) \alpha-\mathrm{TO}^{+}$}

$\mathrm{HF} / 6-31+\mathrm{G}$ *

\# of imaginary frequencies 0

Total energy -690.7353205382

Cartesian coordinates

$\begin{array}{rrrrr}1 & \mathrm{O} & 3.832877 & -.922628 & .068569 \\ 2 & \mathrm{C} & 2.715333 & -.522280 & .018777 \\ 3 & \mathrm{C} & .094609 & .358122 & -.050007 \\ 4 & \mathrm{C} & 1.581889 & -1.517128 & -.021159 \\ 5 & \mathrm{C} & 2.429321 & .953754 & -.013089 \\ 6 & \mathrm{C} & 1.157286 & 1.368674 & -.052057 \\ 7 & \mathrm{C} & .315257 & -1.083397 & -.086128 \\ 8 & \mathrm{C} & 1.997415 & -2.957601 & .002226 \\ 9 & \mathrm{C} & 3.620547 & 1.874649 & .006276 \\ 10 & \mathrm{C} & .724190 & 2.814761 & -.078966 \\ 11 & \mathrm{C} & -.898556 & -1.988942 & -.175585 \\ 12 & \mathrm{O} & -1.067759 & .839599 & -.014471 \\ 13 & \mathrm{H} & 2.711416 & -3.122535 & .798720 \\ 14 & \mathrm{H} & 2.499559 & -3.209621 & -.926705 \\ 15 & \mathrm{H} & 1.157794 & -3.620706 & .139271 \\ 16 & \mathrm{H} & 4.537566 & 1.312064 & .077648 \\ 17 & \mathrm{H} & 3.563687 & 2.552120 & .850592 \\ 18 & \mathrm{H} & 3.651700 & 2.469903 & -.899898 \\ 19 & \mathrm{H} & .158725 & 3.067272 & .810814 \\ 20 & \mathrm{H} & .092761 & 3.014493 & -.936472 \\ 21 & \mathrm{H} & 1.580100 & 3.469201 & -.0131706 \\ 22 & \mathrm{H} & -1.111065 & -2.415726 & .798939 \\ 23 & \mathrm{H} & -.680916 & -2.816136 & -.837311 \\ 24 & \mathrm{C} & -2.354298 & .071637 & .021756 \\ 25 & \mathrm{C} & -3.334651 & .977230 & -.702676 \\ 26 & \mathrm{H} & -4.310990 & .505944 & -.713325 \\ 27 & \mathrm{H} & -3.423776 & 1.932390 & -.200637 \\ 28 & \mathrm{H} & -1.958669 & -.644837 & 2.042549 \\ 29 & \mathrm{C} & -2.704942 & -.081668 & 1.495542 \\ 30 & \mathrm{H} & -2.808974 & .889508 & 1.963129 \\ 31 & \mathrm{H} & -3.651787 & -.602104 & 1.583030 \\ 32 & \mathrm{C} & -2.112806 & -1.236278 & -.713229 \\ 33 & \mathrm{H} & -2.999990 & -1.852293 & -.0627374 \\ 34 & \mathrm{H} & -1.975065 & -1.025365 & -1.769643 \\ 35 & \mathrm{H} & -3.027789 & 1.147923 & -1.727401 \\ & & & & \end{array}$




\section{2) $\left(\mathrm{CH}_{3}\right) \alpha-\mathrm{TOH}$}

HF / 6-31G*

\# of imaginary frequencies 0

Total energy -691.5325251617

Cartesian coordinates

$\begin{array}{rrrrr}1 & \mathrm{O} & 3.722906 & -1.243698 & .062725 \\ 2 & \mathrm{C} & 2.480679 & -.670929 & -.009643 \\ 3 & \mathrm{C} & -.032893 & .424106 & -.181611 \\ 4 & \mathrm{C} & 1.387262 & -1.515587 & -.080207 \\ 5 & \mathrm{C} & 2.343400 & .716534 & -.047009 \\ 6 & \mathrm{C} & 1.071804 & 1.275423 & -.102625 \\ 7 & \mathrm{C} & .110752 & -.955122 & -.189860 \\ 8 & \mathrm{C} & 1.572807 & -3.015851 & -.052299 \\ 9 & \mathrm{C} & 3.589289 & 1.577622 & -.022059 \\ 10 & \mathrm{C} & .869497 & 2.775745 & -.094031 \\ 11 & \mathrm{C} & -1.113689 & -1.841498 & -.294434 \\ 12 & \mathrm{O} & -1.251038 & 1.026728 & -.282073 \\ 13 & \mathrm{H} & 2.586100 & -3.273152 & .214361 \\ 14 & \mathrm{H} & 1.363274 & -3.456312 & -1.024116 \\ 15 & \mathrm{H} & .903392 & -3.481957 & .663854 \\ 16 & \mathrm{H} & 3.967357 & 1.719063 & .990339 \\ 17 & \mathrm{H} & 3.405457 & 2.560206 & -.432178 \\ 18 & \mathrm{H} & 4.380641 & 1.128781 & -.612457 \\ 19 & \mathrm{H} & -.165175 & 3.020898 & .084475 \\ 20 & \mathrm{H} & 1.153248 & 3.219568 & -1.045114 \\ 21 & \mathrm{H} & 1.466731 & 3.248423 & .678034 \\ 22 & \mathrm{H} & -1.293202 & -2.334678 & .6558274 \\ 23 & \mathrm{H} & -.935686 & -2.636017 & -1.011261 \\ 24 & \mathrm{C} & -2.432532 & .292004 & .004413 \\ 25 & \mathrm{C} & -3.566920 & 1.142521 & -.554670 \\ 26 & \mathrm{H} & -4.526000 & .667706 & -.373808 \\ 27 & \mathrm{H} & -3.573539 & 2.119573 & -.084353 \\ 28 & \mathrm{H} & -1.733430 & -.373135 & 1.958881 \\ 29 & \mathrm{C} & -2.580452 & .140036 & 1.520154 \\ 30 & \mathrm{H} & -2.651283 & 1.118258 & 1.982942 \\ 31 & \mathrm{H} & -3.479132 & -.418718 & 1.762779 \\ 32 & \mathrm{C} & -2.349401 & -1.051040 & -.717932 \\ 33 & \mathrm{H} & -3.250098 & -1.629565 & -.537506 \\ 34 & \mathrm{H} & -2.302428 & -.851687 & -1.784480 \\ 35 & \mathrm{H} & -3.444874 & 1.281344 & -1.622786 \\ 36 & \mathrm{H} & 4.307176 & -.705593 & .575278\end{array}$




\section{3) $\left(\mathrm{CH}_{3}\right) \alpha-\mathrm{TO}^{+}$}

HF / 6-31G*

\# of imaginary frequencies 0

Total energy -690.7270133116

Cartesian coordinates

$\begin{array}{rrrrr}1 & \mathrm{O} & 3.832730 & -.923076 & .068953 \\ 2 & \mathrm{C} & 2.715373 & -.522279 & .018322 \\ 3 & \mathrm{C} & .094016 & .358377 & -.051492 \\ 4 & \mathrm{C} & 1.581546 & -1.516930 & -.021575 \\ 5 & \mathrm{C} & 2.429395 & .953817 & -.014662 \\ 6 & \mathrm{C} & 1.157138 & 1.368703 & -.052932 \\ 7 & \mathrm{C} & .314878 & -1.083231 & -.087036 \\ 8 & \mathrm{C} & 1.997875 & -2.957215 & .002988 \\ 9 & \mathrm{C} & 3.620722 & 1.874661 & .004409 \\ 10 & \mathrm{C} & .724094 & 2.814861 & -.077454 \\ 11 & \mathrm{C} & -.898957 & -1.989185 & -.174530 \\ 12 & \mathrm{O} & -1.068444 & .839730 & -.016567 \\ 13 & \mathrm{H} & 2.707931 & -3.121973 & .803117 \\ 14 & \mathrm{H} & 2.505496 & -3.207613 & -.923289 \\ 15 & \mathrm{H} & 1.158267 & -3.621406 & .135018 \\ 16 & \mathrm{H} & 4.538341 & 1.311207 & .059373 \\ 17 & \mathrm{H} & 3.572092 & 2.540588 & .858376 \\ 18 & \mathrm{H} & 3.642382 & 2.482042 & -.893685 \\ 19 & \mathrm{H} & .150863 & 3.062659 & .808523 \\ 20 & \mathrm{H} & .099702 & 3.018015 & -.939344 \\ 21 & \mathrm{H} & 1.580303 & 3.469776 & -.119826 \\ 22 & \mathrm{H} & -1.110559 & -2.414615 & .800670 \\ 23 & \mathrm{H} & -.681818 & -2.817001 & -.835536 \\ 24 & \mathrm{C} & -2.355182 & .071145 & .021299 \\ 25 & \mathrm{C} & -3.335067 & .975774 & -.704419 \\ 26 & \mathrm{H} & -4.311037 & .503770 & -.717637 \\ 27 & \mathrm{H} & -3.425841 & 1.930446 & -.0201806 \\ 28 & \mathrm{H} & -1.961080 & -.648731 & 2.040802 \\ 29 & \mathrm{C} & -2.704167 & -.079773 & 1.495518 \\ 30 & \mathrm{H} & -2.799684 & .892197 & 1.963052 \\ 31 & \mathrm{H} & -3.654761 & -.593034 & 1.584709 \\ 32 & \mathrm{C} & -2.113672 & -1.237752 & -.711964 \\ 33 & \mathrm{H} & -3.000918 & -1.853604 & -.624725 \\ 34 & \mathrm{H} & -1.976344 & -1.027936 & -1.768549 \\ 35 & \mathrm{H} & -3.025610 & 1.147599 & -1.728110\end{array}$




\section{4) $\left(\mathrm{CH}_{3}\right) \alpha-\mathrm{TOH}$}

EDF $2 / 6-31+G$ *

\# of imaginary frequencies 0

Total energy -695.4967740770

Cartesian coordinates

$\begin{array}{rrrrr}1 & \mathrm{O} & 3.654888 & -0.798379 & 0.126301 \\ 2 & \mathrm{C} & 2.336335 & -0.414484 & 0.026535 \\ 3 & \mathrm{C} & -0.279422 & 0.450461 & -0.137584 \\ 4 & \mathrm{C} & 1.312167 & -1.362712 & -0.028822 \\ 5 & \mathrm{C} & 2.081161 & 0.960110 & -0.009967 \\ 6 & \mathrm{C} & 0.754857 & 1.398006 & -0.079388 \\ 7 & \mathrm{C} & -0.019293 & -0.924936 & -0.135247 \\ 8 & \mathrm{C} & 1.674460 & -2.824884 & 0.034929 \\ 9 & \mathrm{C} & 3.217912 & 1.942818 & 0.037359 \\ 10 & \mathrm{C} & 0.441584 & 2.868328 & -0.104974 \\ 11 & \mathrm{C} & -1.164408 & -1.904433 & -0.249888 \\ 12 & \mathrm{O} & -1.552187 & 0.960392 & -0.231399 \\ 13 & \mathrm{H} & 2.241292 & -3.062715 & 0.946033 \\ 14 & \mathrm{H} & 2.283608 & -3.141344 & -0.824306 \\ 15 & \mathrm{H} & 0.795102 & -3.469006 & 0.045387 \\ 16 & \mathrm{H} & 4.177878 & 1.439089 & -0.077285 \\ 17 & \mathrm{H} & 3.240022 & 2.486624 & 0.990734 \\ 18 & \mathrm{H} & 3.126773 & 2.695491 & -0.753505 \\ 19 & \mathrm{H} & -0.625969 & 3.044423 & 0.023351 \\ 20 & \mathrm{H} & 0.741255 & 3.324504 & -1.057714 \\ 21 & \mathrm{H} & 0.979450 & 3.404888 & 0.684279 \\ 22 & \mathrm{H} & -1.311317 & -2.430138 & 0.704467 \\ 23 & \mathrm{H} & -0.922664 & -2.682606 & -0.982528 \\ 24 & \mathrm{C} & -2.667141 & 0.099701 & 0.067358 \\ 25 & \mathrm{C} & -3.881344 & 0.844774 & -0.466922 \\ 26 & \mathrm{H} & -4.798289 & 0.284375 & -0.258640 \\ 27 & \mathrm{H} & -3.963715 & 1.828609 & 0.004670 \\ 28 & \mathrm{H} & -1.863428 & -0.526577 & 1.998942 \\ 29 & \mathrm{C} & -2.769827 & -0.079935 & 1.582465 \\ 30 & \mathrm{H} & -2.917734 & 0.891364 & 2.063662 \\ 31 & \mathrm{H} & -3.618236 & -0.723777 & 1.838285 \\ 32 & \mathrm{C} & -2.462407 & -1.220349 & -0.669947 \\ 33 & \mathrm{H} & -3.318648 & -1.881395 & -0.497564 \\ 34 & \mathrm{H} & -2.433987 & -1.000457 & -1.743698 \\ 35 & \mathrm{H} & -3.798621 & 0.989752 & -1.548159 \\ 36 & \mathrm{H} & 3.717819 & -1.761987 & 0.088945\end{array}$




\section{5) $\left(\mathrm{CH}_{3}\right) \alpha-\mathrm{TO}^{+}$}

EDF $2 / 6-31+G$ *

\# of imaginary frequencies 0

Total energy -694.6367526139

Cartesian coordinates

\begin{tabular}{|c|c|c|c|c|}
\hline 1 & 0 & 3.570463 & -0.894401 & 0.079019 \\
\hline 2 & $\mathrm{C}$ & 2.425304 & -0.485395 & 0.027343 \\
\hline 3 & $\mathrm{C}$ & -0.199050 & 0.418354 & -0.03727 \\
\hline 4 & $\mathrm{C}$ & 1.292841 & -1.470260 & -0.00701 \\
\hline 5 & $\mathrm{C}$ & 2.149723 & 0.986444 & -0.00739 \\
\hline 6 & $\mathrm{C}$ & 0.859622 & 1.415242 & -0.03736 \\
\hline 7 & $\mathrm{C}$ & 0.014576 & -1.012512 & -0.06156 \\
\hline 8 & $\mathrm{C}$ & 1.641265 & -2.915631 & 0.00436 \\
\hline 9 & $\mathrm{C}$ & 3.333601 & 1.891766 & 0.00730 \\
\hline 10 & $\mathrm{C}$ & 0.456446 & 2.857895 & -0.05312 \\
\hline 11 & $\mathrm{C}$ & -1.179185 & -1.924187 & -0.13285 \\
\hline 12 & 0 & -1.385136 & 0.923031 & -0.01308 \\
\hline 13 & $\mathrm{H}$ & 2.696655 & -3.059855 & 0.23353 \\
\hline 14 & $\mathrm{H}$ & 1.447656 & -3.364713 & -0.97952 \\
\hline 15 & $\mathrm{H}$ & 1.032002 & -3.466161 & 0.72815 \\
\hline 16 & $\mathrm{H}$ & 4.257994 & 1.315478 & 0.01793 \\
\hline 17 & $\mathrm{H}$ & 3.321816 & 2.542412 & 0.88924 \\
\hline 18 & $\mathrm{H}$ & 3.340389 & 2.545476 & -0.87199 \\
\hline 19 & $\mathrm{H}$ & -0.125012 & 3.114153 & 0.83868 \\
\hline 20 & $\mathrm{H}$ & -0.176821 & 3.082461 & -0.91708 \\
\hline 21 & $\mathrm{H}$ & 1.326932 & 3.510738 & -0.08912 \\
\hline 22 & $\mathrm{H}$ & -1.386115 & -2.337501 & 0.86288 \\
\hline 23 & $\mathrm{H}$ & -0.943170 & -2.782918 & -0.76750 \\
\hline 24 & $\mathrm{C}$ & -2.663202 & 0.119430 & 0.02405 \\
\hline 25 & $\mathrm{C}$ & -3.652504 & 0.996099 & -0.71802 \\
\hline 26 & $\mathrm{H}$ & -4.634980 & 0.515691 & -0.70719 \\
\hline 27 & $\mathrm{H}$ & -3.748049 & 1.973143 & -0.23824 \\
\hline 28 & $\mathrm{H}$ & -2.262124 & -0.572992 & 2.06002 \\
\hline 29 & $\mathrm{C}$ & -3.012125 & -0.009843 & 1.49902 \\
\hline 30 & $\mathrm{H}$ & -3.117842 & 0.976243 & 1.95754 \\
\hline 31 & $\mathrm{H}$ & -3.969556 & -0.530025 & 1.59476 \\
\hline 32 & $\mathrm{C}$ & -2.403770 & -1.200097 & -0.68208 \\
\hline 33 & $\mathrm{H}$ & -3.294255 & -1.826322 & -0.57860 \\
\hline 34 & $\mathrm{H}$ & -2.274304 & -1.008926 & -1.75359 \\
\hline 35 & $\mathrm{H}$ & -3.355078 & 1.140761 & -1.75977 \\
\hline
\end{tabular}

